\title{
Organic anion transporters OAT1 and OAT4 mediate the high affinity transport of glutarate derivatives accumulating in patients with glutaric acidurias
}

\author{
Yohannes Hagos • Wolfgang Krick • Thomas Braulke • \\ Chris Mühlhausen - Gerhard Burckhardt • \\ Birgitta C. Burckhardt
}

Received: 18 December 2007 / Revised: 8 February 2008 / Accepted: 29 February 2008 / Published online: 26 March 2008

(C) The Author(s) 2008

\begin{abstract}
Glutaric acidurias are rare inherited neurodegenerative disorders accompanied by accumulation of the metabolites glutarate (GA) and 3-hydroxyglutarate (3OHGA), glutaconate, L-, or D-2-hydroxyglutarate (L-2OHGA, D-2OHGA) in all body fluids. Oocytes expressing the human (h) sodium-dicarboxylate cotransporter $(\mathrm{NaDC} 3)$ showed sodium-dependent inward currents mediated by GA, 3OHGA, L-, and D-2OHGA. The organic anion transporters (OATs) were examined as additional transporters for GA derivatives. The uptake of $\left[{ }^{3} \mathrm{H}\right] p$-aminohippurate in hOAT1-transfected human embryonic kidney (HEK293) cells was inhibited by GA, 3OHGA, D-, or L-2OHGA in a concentration-dependent manner. None of these compounds affected the hOAT3-mediated uptake of $\left[{ }^{3} \mathrm{H}\right]$ estrone sulfate (ES). In hOAT4-expressing cells and oocytes, ES uptake was strongly increased by intracellular GA derivatives. The data provide a model for the concerted action of OAT1 and $\mathrm{NaDC} 3$ mediating the basolateral uptake, and OAT4 mediating apical secretion of GA derivatives from proximal tubule cells and therefore contribute to the renal clearance of these compounds.
\end{abstract}

Y. Hagos • W. Krick • G. Burckhardt • B. C. Burckhardt $(\bowtie)$

Zentrum Physiologie und Pathophysiologie,

Abt. Vegetative Physiologie und Pathophysiologie,

Universitätsmedizin Göttingen,

Humboldtallee 23,

37073 Göttingen, Germany

e-mail: bcburckhardt@physiol.med.uni-goettingen.de

T. Braulke $\cdot$ C. Mühlhausen

Kinderklinik-Biochemie, Campus Forschung,

Universitätsklinikum Hamburg-Eppendorf,

Martinistrasse 52,

20246 Hamburg, Germany
Keywords $\mathrm{NaDC} 3 \cdot$ Organic anion transporters · OAT1 . OAT4 - Renal excretion of glutarate derivatives .

Sodium-dependent dicarboxylate transporters

\section{Introduction}

Several inborn metabolic diseases lead to the accumulation of dicarboxylic acids such as glutaric acid. Glutaric aciduria type 1 (GA1) is caused by deficiency of glutaryl-CoA dehydrogenase (EC 1.3.99.7; GCDH), a mitochondrial enzyme involved in the degradation of tryptophan and lysine. Defective GCDH causes insufficient glutaryl-CoA breakdown and results in an accumulation of glutaric acid (GA), 3-hydroxyglutaric acid (3OHGA), glutaconate (GC), and glutarylcarnitine in tissues, plasma, cerebrospinal fluid, and urine [1-4]. During catabolic situations, GA1 patients are subject to the development of encephalopathic crises, leading to striatal degeneration with a subsequent irreversible dystonic-dyskinetic movement disorder. Histopathological findings include neuronal loss and astrogliosis in the striatum, subdural hemorrhages, and spongiform vacuolation of the white matter. These symptoms and the associated histopathological changes in putamen and caudatum mainly occur between the 3rd and 36th month of age triggered by a nonspecific disease like fever, respiratory infection, or diarrhea [1-5].

Glutaric aciduria type 2 (GA2) is an autosomal recessive disorder associated with hypoketotic hypoglycemia, metabolic acidosis, liver-, kidney-, and cardiomegaly, often leading to an intractable neonatal illness with early death. GA2 is caused by a defect of electron transfer flavoprotein (ETF) or ETF cytochrome Q oxidoreductase and subsequent deficiencies of multiple acyl-CoA dehydrogenases. 
Defective fatty acid oxidation as well as amino acid degradation led to accumulation of GA, lactate, and dicarboxylic acids such as ethylmalonate and adipate [6]. Two other clinical entities, both affecting the nervous system, are characterized by accumulation of D- and L-2-hydroxyglutaric acids (D-2OHGA, L-2OHGA), respectively, in tissues and body fluids [7-9]. Patients with D-2-hydroxyglutaric aciduria suffer from developmental delay, macroencephalus, epilepsy, and cerebral atrophy. Defects of either hydroxyacid-oxoacid transhydrogenase or D-2OHGA dehydrogenase have been observed in these patients. L-2-hydoxyglutaric aciduria is caused by deficiency of the FAD-dependent L-2-hydroxyglutarate dehydrogenase and accompanied by psychomotor retardation, ataxia, hypotonia, tremor, and seizures [10].

For diagnosis, newborn screening programs have been developed based mainly on the detection of glutarylcarnitine in dried blood spots [11]. GA1 is considered treatable if diagnosed before onset of encephalopathic crises. Patients excrete with the urine large amounts of GA and 3OHGA which exceeds glomerular filtration rate, indicating that these substances have to be secreted [1].

At physiological $\mathrm{pH}$, GA derivatives exist as highly hydrophilic dicarboxylate anions requiring specific transport systems to translocate these compounds across biological membranes. Recently, we showed that GA, $\alpha$-ketoglutarate $(\alpha \mathrm{KG})[12]$, and 3OHGA [13] are substrates of the sodium dicarboxylate cotransporter $3(\mathrm{NaDC} 3$, aliases $\mathrm{NaC} 3$, SDCT2 [14]). Further renal transporters interacting with $\alpha \mathrm{KG}$ and GA are the organic anion transporters (OAT) 1 and OAT3 located in the basolateral membrane and OAT4 in the apical membrane of proximal tubular cells [15-17]. OATs are in the focus of medical interest due to their roles in the excretion of many clinically drugs such as antibiotics, antivirals, $\mathrm{H}_{2}$ antagonists, antihypertensives, cytostatics, diuretics, nonsteroidal anti-inflammatory drugs, statins, and uricosurics [15-17]. In the present study, we report on the capability of OATs to transport GA derivatives across membranes. Since these transporters are present also in the brain [18], OATs may not only influence plasma levels of GA derivatives by securing their renal excretion but may also have an important impact on their levels in brain tissue.

\section{Materials and methods}

Solutions A standard oocyte Ringer solution (ORi) was used for oocyte preparation, storage, and for the uptake as well as for the electrophysiological measurements. ORi contained (in $\mathrm{mM}$ ): $110 \mathrm{NaCl}$, three $\mathrm{KCl}$, two $\mathrm{CaCl}_{2}$, five 4-(2-hydroxyethyl)-1-piperazineethanesulfonic acid (HEPES)/Tris adjusted to $\mathrm{pH}$ 7.5. All chemicals, including those for ORi, for oocyte preparation and storage as well as for the electrophysiological experiments on oocytes and the uptake experiments on oocytes and HEK293 cells were purchased from Sigma (Taufkirchen, Germany). 3OHGA and $\left[{ }^{3} \mathrm{H}\right] 3 \mathrm{OHGA}$ were kindly provided by J. Thiem (Institute of Organic Chemistry, University of Hamburg, Hamburg, Germany). Their synthesis was described in the additional material provided electronically in [13]. Radioactively labeled $\left[{ }^{3} \mathrm{H}\right]$ estrone sulfate $(\mathrm{ES}, 43.5 \mathrm{Ci} / \mathrm{mmol})$ and $\left[{ }^{3} \mathrm{H}\right]$ para-aminohippurate (PAH, $4.18 \mathrm{Ci} / \mathrm{mmol}$ ) were from Perkin Elmer Life Sciences (Shelton, CT, USA).

In vitro transcription of $c R N A \mathrm{hNaDC} 3$ and hOAT4 cDNAs were used as templates for cRNA synthesis. Plasmids were linearized with Not I and in vitro cRNA transcription was performed using $\mathrm{T} 7 \mathrm{mMessage}$ mMachine kit (Ambion, Austin, TX, USA) according to the manufacturer's instructions. The resulting cRNA was suspended in purified, RNAse-free water to a final concentration of $1 \mu \mathrm{g} / \mu \mathrm{l}$.

Oocyte preparation and storage Female Xenopus laevis (Nasco, Fort Atkinson, WI, USA) were subjected to partial ovariectomy according to protocols approved by the Universitätsmedizin Göttingen, Medical Center Animal Care and Use Committee. Stage V and VI oocytes were prepared by an overnight treatment in ORi supplemented with collagenase (Typ CLS II, Biochrom, Berlin, Germany). Subsequently, after being washed with $\mathrm{Ca}^{2+}$-free ORi to remove adhering follicle cells, the oocytes were injected with $23 \mathrm{nl}$ of $1 \mu \mathrm{g} / \mu \mathrm{l}$ cRNA coding for $\mathrm{hNaDC} 3$, hOAT4, or water. Afterward, the injected oocytes were incubated at $16^{\circ}-18^{\circ} \mathrm{C}$ in ORi supplemented with gentamycin $(12 \mathrm{mg} /$ $\mathrm{ml}$ ) and $2.5 \mathrm{mM}$ sodium pyruvate. After 3 to 4 days of incubation with daily medium changes, oocytes were used for tracer uptake and electrophysiological studies.

hOAT1-, hOAT3-, and hOAT4-transfected HEK293 cells Human OAT1 and OAT3 (GeneBank accession number: AF097490 and BI760120) were obtained from Resource Center for Genome Research (RZPD, Berlin, Germany). Human OAT4 (GeneBank accession number AL514126) was from Invitrogen (Groningen, The Netherlands). The stably transfected human epithelial kidney cells T-REX ${ }^{\mathrm{TM}}$ HEK293-hOAT1, HEK293-hOAT3, and HEK293-hOAT4 were established by using the Flp- $\operatorname{In}^{\mathrm{TM}}$ expression system (Invitrogen) as previously described [19]. Stably transfected HEK293 cells were selected by hygromycine $(10 \mu \mathrm{g} / \mathrm{ml})$ and grown in flasks in high glucose DMEM medium (Invitrogen) supplemented with 10\% fetal calf serum, 1\% penicillin/streptomycin, and blasticidine (5 mg/l; Sigma). For control, the cells were transfected with the vector alone. Cultures were maintained in humidified atmosphere that contained $5 \% \mathrm{CO}_{2}$ at $37^{\circ} \mathrm{C}$. 
Electrophysiological experiments These studies were carried out 3 days after cRNA injection at room temperature. Oocytes were placed in a $0.5 \mathrm{ml}$ bath chamber on the stage of a dissecting microscope and impaled under direct view with borosilicate glass (BioMedical Instruments, Zöllnitz, Germany) microelectrodes filled with $3 \mathrm{M} \mathrm{KCl}$. Current recordings were made using the two electrode voltage clamp technique in the voltage clamp mode with a commercial amplifier (OC725, Warner, Hambden, CT, USA). Borosilicate glass microelectrodes had resistances of $\sim 1 \mathrm{M} \Omega$.

Tracer uptake experiments HEK293-hOAT1, HEK293hOAT3, HEK293-hOAT4 and vector-transfected control cells were harvested and plated into 24-well plastic dishes (Sarstedt, Nürmbrecht, Germany) at a density of $2 \times 10^{5}$ cells/well. Transport assays were performed $48 \mathrm{~h}$ after seeding in mammalian Ringer solution that contained (in $\mathrm{mM}$ ): 130 $\mathrm{NaCl}$, four $\mathrm{KCl}$, one $\mathrm{CaCl}_{2}$, one $\mathrm{MgSO}_{4}$, one $\mathrm{NaH}_{2} \mathrm{PO}_{4}, 20$ HEPES, and 18 glucose at pH 7.4. The cells were washed twice with $0.5 \mathrm{ml}$ Ringer and incubated for $5 \mathrm{~min}$ in Ringer that contained 10 or $20 \mathrm{nM}\left[{ }^{3} \mathrm{H}\right]$ estrone sulfate for OAT3and OAT4-expressing HEK293 cells, or $1.2 \mu \mathrm{M}\left[{ }^{3} \mathrm{H}\right] \mathrm{PAH}$ or $\left.20 \mathrm{nM} \mathrm{[}{ }^{3} \mathrm{H}\right] 3 \mathrm{OHGA}$ for OAT1-expressing HEK293 cells. Uptake was terminated by removal of the radioactive medium and immediate $3 \times 3 \mathrm{ml}$ washes with ice-cold Ringer solution. The cells were dissolved in $0.5 \mathrm{ml}$ one $\mathrm{N} \mathrm{NaOH}$ by gently shaking for $120 \mathrm{~min}$ followed by neutralization with $0.5 \mathrm{ml}$ one $\mathrm{N} \mathrm{HCl}$. The $\left({ }^{3} \mathrm{H}\right)$ content was determined by liquid scintillation counting (Tricarb 2900TR, Perkin Elmer, Rodgau, Germany).

Some experiments required high intracellular concentrations of the GA derivatives within the cells or the oocytes. For that purpose, OAT4-transfected HEK293 cells were incubated for $2 \mathrm{~h}$ in Ringer containing in addition 1 mM 3OHGA, D-, or L-2OHGA. In a second set, oocytes were injected with $23 \mathrm{nl}$ of either $60 \mathrm{mM} 3 \mathrm{OHGA}$, D-, or L2OHGA or an equivalent amount of ORi (control). Afterward, uptake experiments in cells were performed as described above. The uptake of $\left[{ }^{3} \mathrm{H}\right] \mathrm{ES}$ in oocytes was measured as described previously [20].

Statistics Paired Student's $t$-tests were used to show statistically significant differences of GA derivative-associated inward currents to the currents evoked by GA in the same oocyte. The same test was also used for demonstrating statistical significance of PAH or ES uptake in cis-inhibition or trans-stimulation experiments. Statistical significance was set at $p<0.01$. $K_{\mathrm{i}}$ values were determined by Dixon plot analysis. To estimate the kinetic parameters for the transport by $\mathrm{hNaDC} 3$, the equation $I=I_{\max }[S] /\left(K_{\mathrm{m}}+[S]\right.$ ) (SigmaPlot 10 software, Systat, Point Richmond, CA, USA) was used, where $I$ is the current actually measured, $I_{\max }$ the maximum current, $K_{\mathrm{m}}$ the Michaelis-Menten constant, and $S$ the substrate concentration.

\section{Results}

Substrate specificity for glutarate derivatives and other dicarboxylates of human NaDC3 expressed in $X$. laevis oocytes Perfusion of hNaDC3-expressing oocytes with L-, D-2OHGA, GC, and adipate (each $1 \mathrm{mM}$ ) elicited inward currents (Fig. 1). GA, $\alpha \mathrm{KG}$, and 3OHGA were used as controls [13]. In the absence of sodium, these currents were reduced by $81-99 \%$, indicating their dependence on sodium (Fig. 1). The magnitude of the steady state currents was a saturable function of the concentration of the GA derivatives (Fig. 2). Although the $K_{\mathrm{m}}$ was the lowest for GA and adipate $(0.04 \mathrm{mM})$, the transport capacity $\left(I_{\max }\right)$ for $\mathrm{D}-, \mathrm{L}-$ 2OHGA, and 3OHGA was greater than that for GA and adipate (Table 1). For three distinct voltages $-90,-60$, and $-30 \mathrm{mV}$, the data were fitted to the Michaelis-Menten equation and the $K_{\mathrm{m}}$ values obtained were plotted as a function of membrane potential (Fig. 2, bottom panel, right side). For substitutions at position $\mathrm{C} 2(\alpha \mathrm{KG}, \mathrm{D}-$, and $\mathrm{L}-$ 2OHGA), $K_{\mathrm{m}}$ was unaffected by the potential applied. Substitutions at C3 (3OHGA) or introduction of a double bound (GC) yielded comparably higher $K_{\mathrm{m}}$ values at any membrane potential. Furthermore, $K_{\mathrm{m}}$ values for $3 \mathrm{OHGA}$ and GC increased at depolarizing potentials.

Effect of various GA derivatives on PAH or ES uptake by the human organic anion transporters OAT1, OAT3, and OAT4 In proximal tubule cells, the transport of dicarboxylates is

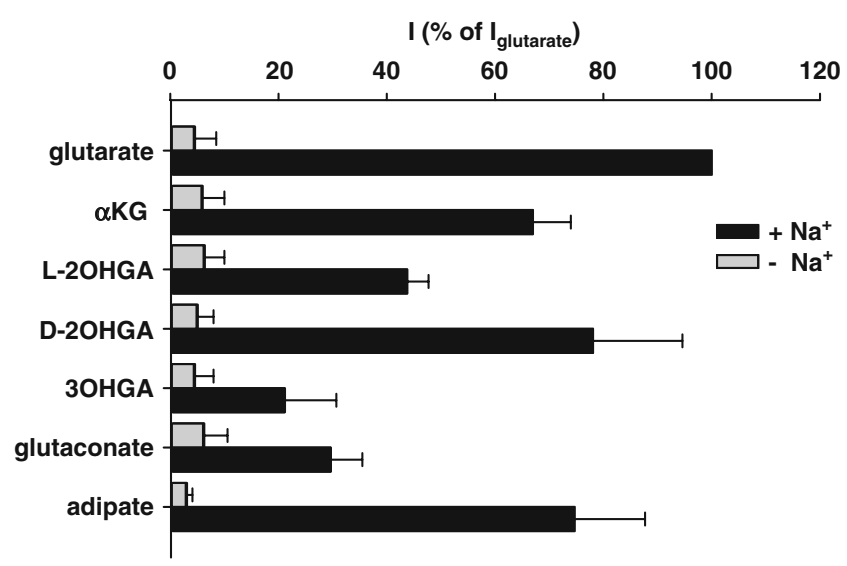

Fig. 1 Inward currents induced by GA derivatives in $\mathrm{NaDC} 3-$ expressing oocytes. At a holding potential of $-60 \mathrm{mV}$, oocytes were superfused with ORi or the indicated substrates $(1 \mathrm{mM})$ in the presence (black columns) or absence (gray columns) of sodium. Substrate-associated currents, $I$, are presented as percent of the current $I$ produced by glutarate in the same batch of oocytes and are shown as mean \pm SEM from $n$ oocytes $/ m$ frogs: GA $17 / 11, \alpha \mathrm{KG} 4 / 3, \mathrm{~L}-2 \mathrm{OHGA}$ $4 / 3$, D-2OHGA $4 / 3$, 3OHGA $8 / 4$, glutaconate $4 / 3$, and adipate $4 / 3$ 
Fig. 2 Effect of membrane potential on substrate-associated inward currents and affinities of NaDC3. Steady state substrateassociated currents were measured as a function of three different membrane potentials ( $V_{\mathrm{c}}:-90$ (filled square), -60 (filled circle), -30 (filled triangle) $\mathrm{mV}$ ) using each of the indicated substrate and five individual concentrations between 0.01 and $5 \mathrm{mM}$. $K_{\mathrm{m}}$ was obtained by fitting $I=I_{\max }[S] /$ $\left(K_{\mathrm{m}}+[S]\right)$ to the data. The results represent the mean \pm SEM from $n$ oocytes $/ m$ frogs. The last graph shows the calculated $K_{\mathrm{m}}$ values. For the GA derivatives, the following symbols are used: (4/3, unfilled square) glutaconate; $(7 / 5$, filled inverted triangle) 3OHGA; (5/3, unfilled circle) glutarate and $(3 / 3)$ adipate; $(4 / 4$, filled circle) $\alpha \mathrm{KG}$; (5/ 5 , unfilled triangle) L-2OHGA; $(3 / 3$, filled circle) D-2OHGA
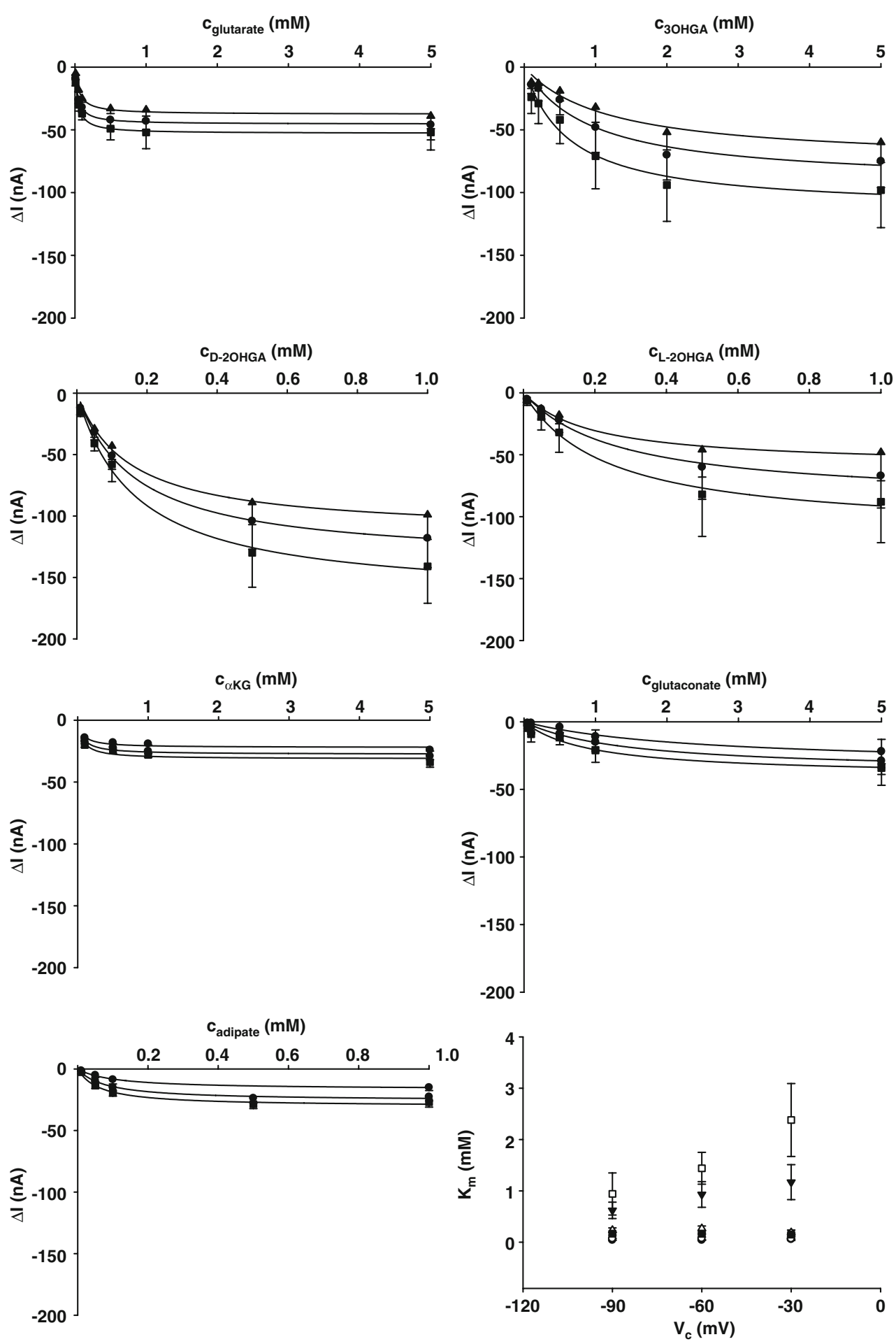

tightly coupled to the transport of organic anions via the organic anion transporters OAT1, OAT3, and OAT4 [15, 16]. Therefore, the impact of GA derivatives on OATmediated substrate transfer was investigated. HEK293 cells transfected with the human OAT1, OAT3, or OAT4 cDNA exhibited different inhibition patterns upon simultaneous application of their prototypical substrate and GA derivatives, adipate, or glutamate. Whereas $\left[{ }^{3} \mathrm{H}\right] \mathrm{PAH}$ uptake in hOAT1-transfected HEK293 cells was inhibited by more than $80 \%$ of $0.1 \mathrm{mM} \mathrm{GA}, \alpha \mathrm{KG}$, or adipate (Fig. 3a), these compounds were less effective on hOAT3-mediated $\left[{ }^{3} \mathrm{H}\right] \mathrm{ES}$ uptake (Fig. 3b). 3OHGA, D-, and L-2OHGA inhibited $\left[{ }^{3} \mathrm{H}\right]$ 
Table 1 Kinetic parameters for NaDC3 substrates

\begin{tabular}{lcccc}
\hline Compound & $\mathrm{K}_{\mathrm{m}}(\mathrm{mM})$ & \multicolumn{1}{l}{$\begin{array}{l}\mathrm{I}_{\max } \\
(\mathrm{nA})\end{array}$} & $\begin{array}{l}\mathrm{I}_{\max } / \mathrm{K}_{\mathrm{m}} \\
(\mathrm{nA} / \mathrm{mM})\end{array}$ & $n / m$ \\
\hline Glutarate & $0.040 \pm 0.02$ & $-45 \pm 1$ & $1125 \pm 37$ & $5 / 3$ \\
$\alpha \mathrm{KG}$ & $0.072 \pm 0.027$ & $-28 \pm 2$ & $452 \pm 135$ & $4 / 4$ \\
L-2OHGA & $0.267 \pm 0.048$ & $-87 \pm 5$ & $336 \pm 48$ & $5 / 5$ \\
D-2OHGA & $0.164 \pm 0.014$ & $-138 \pm 4$ & $847 \pm 58$ & $3 / 3$ \\
3OHGA & $0.93 \pm 0.25$ & $-93 \pm 9$ & $107 \pm 23$ & $7 / 5$ \\
Glutaconate & $1.44 \pm 0.31$ & $-37 \pm 3$ & $26 \pm 4$ & $4 / 3$ \\
Adipate & $0.040 \pm 0.003$ & $-45 \pm 1$ & $1129 \pm 82$ & $3 / 3$ \\
\hline
\end{tabular}

Values are means \pm SEM from $n$ oocytes of $m$ donors. $K_{\mathrm{m}}$ (Michaelis constant) is the substrate concentration at half-maximal current; $I_{\max }$ is the maximum current observed at saturating substrate concentrations at a holding potential of $-60 \mathrm{mV}$.

PAH uptake in OAT1-expressing cells significantly (Fig. 3a), but showed no effects on OAT3-mediated $\left[{ }^{3} \mathrm{H}\right] \mathrm{ES}$ uptake (Fig. 3b). All compounds failed to impair the $\left[{ }^{3} \mathrm{H}\right] \mathrm{ES}$ uptake in OAT4-expressing HEK293 cells (Fig. 3c). The dicarboxylic amino acid glutamate $(0.1 \mathrm{mM})$ did neither inhibit $\left[{ }^{3} \mathrm{H}\right]$ PAH nor $\left[{ }^{3} \mathrm{H}\right] \mathrm{ES}$ uptake in cells transfected with OAT1, OAT3, or OAT4 cDNA, respectively.

Inhibition of OAT1-mediated PAH uptake by 3OHGA, D-, and $L-2 O H G A$ To analyze the kinetics of the inhibitory effect of GA derivatives on hOAT1, Dixon plots were used. In three independent experiments, a competitive inhibition of PAH uptake by 3OHGA, D-2OHGA, and L-2OHGA was observed (Fig. 4a,b,c) showing a $K_{\mathrm{i}}$ of $0.098 \pm 0.030 \mathrm{mM}$, $0.369 \pm 0.081 \mathrm{mM}$, and $0.748 \pm 0.032 \mathrm{mM}$, respectively.

Direct evidence of transport of $3 O H G A, D-$, and $L-2 O H G A$ by hOATl-transfected HEK293 cells Since 3OHGA $(0.1 \mathrm{mM})$ inhibited PAH uptake competitively, transstimulation experiments with the GA derivatives on OAT1-transfected HEK293 cells were performed (Fig. 5a). All three GA derivatives increased $\left[{ }^{3} \mathrm{H}\right] \mathrm{PAH}$ uptake indicating exchange of PAH with either $3 \mathrm{OHGA}, \mathrm{D}-$, or L2OHGA. 3OHGA which showed the lowest $K_{\mathrm{i}}$ in the Dixon plots (Fig. 4a), evoked the largest trans-stimulation. In addition, transport of $\left[{ }^{3} \mathrm{H}\right] 3 \mathrm{OHGA}(20 \mathrm{nM})$ in OAT1transfected HEK293 cells was assayed. The uptake of $\left[{ }^{3} \mathrm{H}\right]$ 3 OHGA measured within $5 \mathrm{~min}$ was $3.8 \pm 1.6 \mathrm{pmol} / 5 \mathrm{~min} \times$ $2 \times 10^{5}$ cells in hOAT1-transfected and $1.2 \pm 0.1 \mathrm{pmol} /$ $5 \min \times 2 \times 10^{5}$ cells in vector-transfected cells (Fig. $5 \mathrm{~b}$ ). Despite the low concentration of labeled 3OHGA used, uptake of 3OHGA was enhanced 3.1 \pm 1.4 times in OAT1transfected over vector-transfected cells.

Trans-stimulation of ES uptake by $3 O H G A$ Because OAT4 accepted GA only from the intracellular compartment [20], OAT4-transfected HEK293 cells were preincubated for $2 \mathrm{~h}$

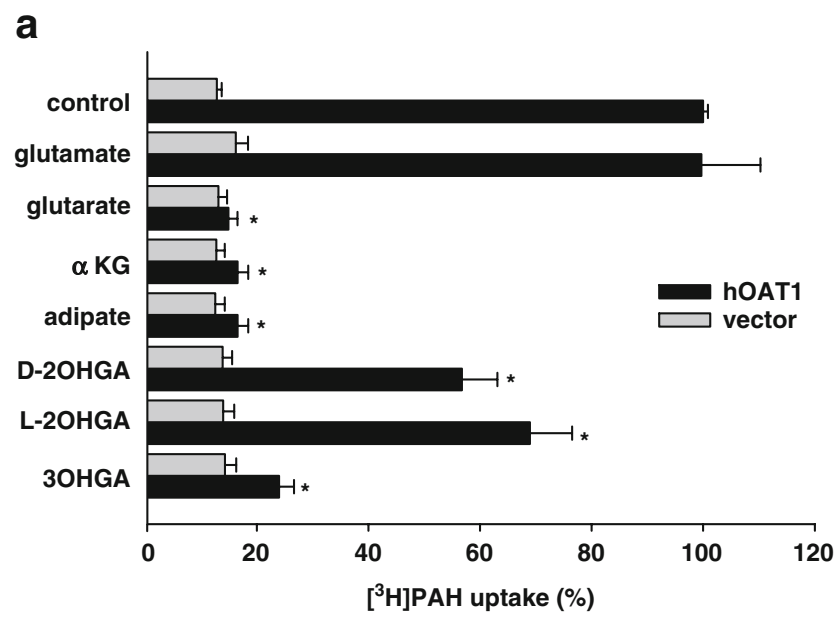

b

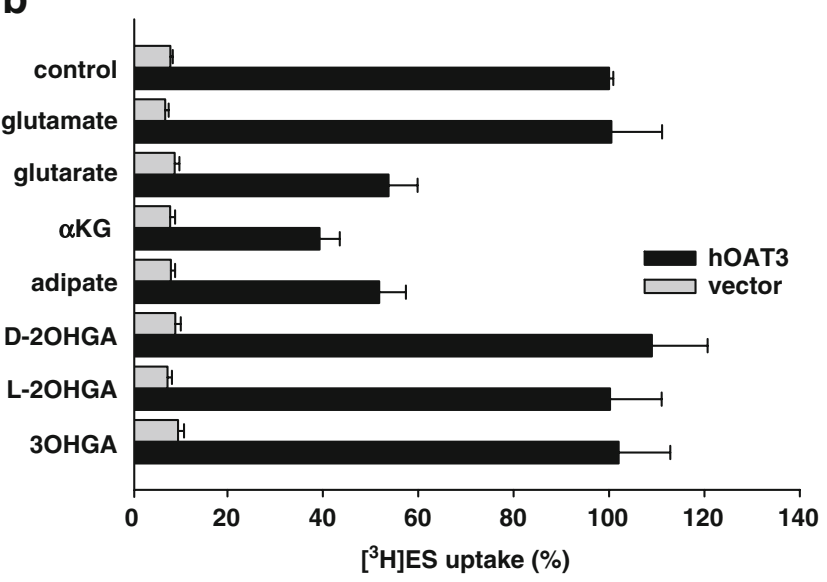

C

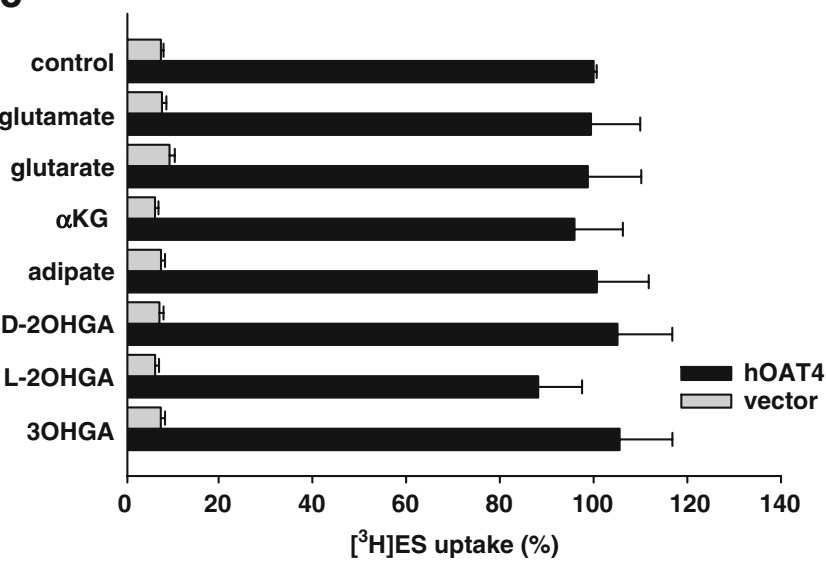

Fig. 3 Cis-inhibitory effects of dicarboxylates on uptake of $\left[{ }^{3} \mathrm{H}\right] \mathrm{PAH}$ and $\left[{ }^{3} \mathrm{H}\right]$ ES in HEK293 cells expressing OAT1 (a), OAT3 (b), or OAT4 (c). The study was performed in Ringer solution without or with $0.1 \mathrm{mM}$ of the indicated dicarboxylates. The concentrations of labeled substrates were $1.2 \mu \mathrm{M}\left[{ }^{3} \mathrm{H}\right] \mathrm{PAH}(\mathbf{a})$ and $10 \mathrm{nM}\left[{ }^{3} \mathrm{H}\right] \mathrm{ES}(\mathbf{b}, \mathbf{c})$. The gray columns represent the uptake in vector-transfected cells, and the black columns represent OAT1-, OAT3-, or OAT4-expressing HEK293 cells. All experiments were standardized by setting the control (without inhibitor) of each experiment to $100 \%$. The values represent the mean \pm SEM of three independent experiments with four repeats each. Significance calculated by Student's $t$-test relative to Ringer solution was accepted at $p<0.01\left(^{*}\right)$ 
Fig. 4 Dixon plot analysis for 3OHGA (a), D-2OHGA (b), and L2OHGA (c) on OAT1-mediated PAH uptake. OAT1-transfected HEK293 cells were incubated in $10 \mu \mathrm{M}$ (filled circle; $1.2 \mu \mathrm{M}\left[{ }^{3} \mathrm{H}\right]$ PAH plus $8.8 \mu \mathrm{M}$ unlabeled PAH) or $100 \mu \mathrm{M}$ (unfilled circle; $1.2 \mu \mathrm{M}$ $\left[{ }^{3} \mathrm{H}\right] \mathrm{PAH}$ plus $98.8 \mu \mathrm{M}$ unlabeled $\left.\mathrm{PAH}\right) \mathrm{PAH}$ in the absence or presence of dicarboxylates at the indicated concentrations. Each plot shows a representative experiment out of a series of four independent experiments for each experimental condition

in solutions containing either $1 \mathrm{mM} 3 \mathrm{OHGA}$, D-, or L2OHGA to increase the intracellular concentration of these compounds. Subsequent measurement of ES uptake for 5 min resulted in a trans-stimulation of $\left[{ }^{3} \mathrm{H}\right] \mathrm{ES}$ uptake (Fig. 6a) by a factor of $1.44 \pm 0.03,1.54 \pm 0.02,1.73 \pm 0.02$, indicating an exchange of 3OHGA, D-, and L-2OHGA present within the cell against ES from the bath.

OAT4-expressing oocytes were injected with 3OHGA, L-, and D-2OHGA to efficiently increase the intracellular concentration of these compounds. When ES uptake in buffer-injected oocytes was set to $100 \%$, ES uptake was increased by $3 \mathrm{OHGA}$, L-, and D-2OHGA by a factor of $1.82 \pm 0.07,2.38 \pm 0.26$, and $2.11 \pm 0.21$, respectively (Fig. 6b).

\section{Discussion}

GA1 is caused by a defect in the mitochondrial glutarylCoA dehydrogenase. Individuals suffering from GA1 exhibit high concentrations of GA and 3OHGA in their body fluids and tissues. An additional increase in plasma and brain concentrations of these metabolites during catabolic crises is thought to be responsible for acute striatal degeneration. Prerequesite for the cytotoxic effects of GA and 3OHGA on brain and vascular cells $[4,21]$ is the transport of metabolites across various biological membranes, including mitochondrial and plasma membranes and trans-endothelial transport.

Functional investigations in vitro recently demonstrated the capability of the NaDC3 to translocate the GA1-specific metabolite 3OHGA with low affinity [13]. Here, we show that also L-, D-2OHGA, and GC evoked sodium-dependent inward currents in $X$. laevis oocytes expressing hNaDC3. Additionally, adipate, a metabolite accumulating in GA2, showed a sodium-dependent inward current. The affinities of $\mathrm{hNaDC} 3$ for these dicarboxylates decreased in the order $\mathrm{GA}=$ adipate $>\alpha \mathrm{KG}>\mathrm{D}-2 \mathrm{OHGA}>\mathrm{L}-2 \mathrm{OHGA}>3 \mathrm{OHGA}>$ GC. This sequence indicates that $\mathrm{hNaDC} 3$ binds preferentially unsubstituted dicarboxylates with five (GA) and six carbon (adipate) backbones. A double bond within the backbone as in GC largely decreased the affinity, suggesting that the carbon backbone must be highly flexible for interaction with hNaDC3. Substitutions at $\mathrm{C} 2(\alpha \mathrm{KG}$,
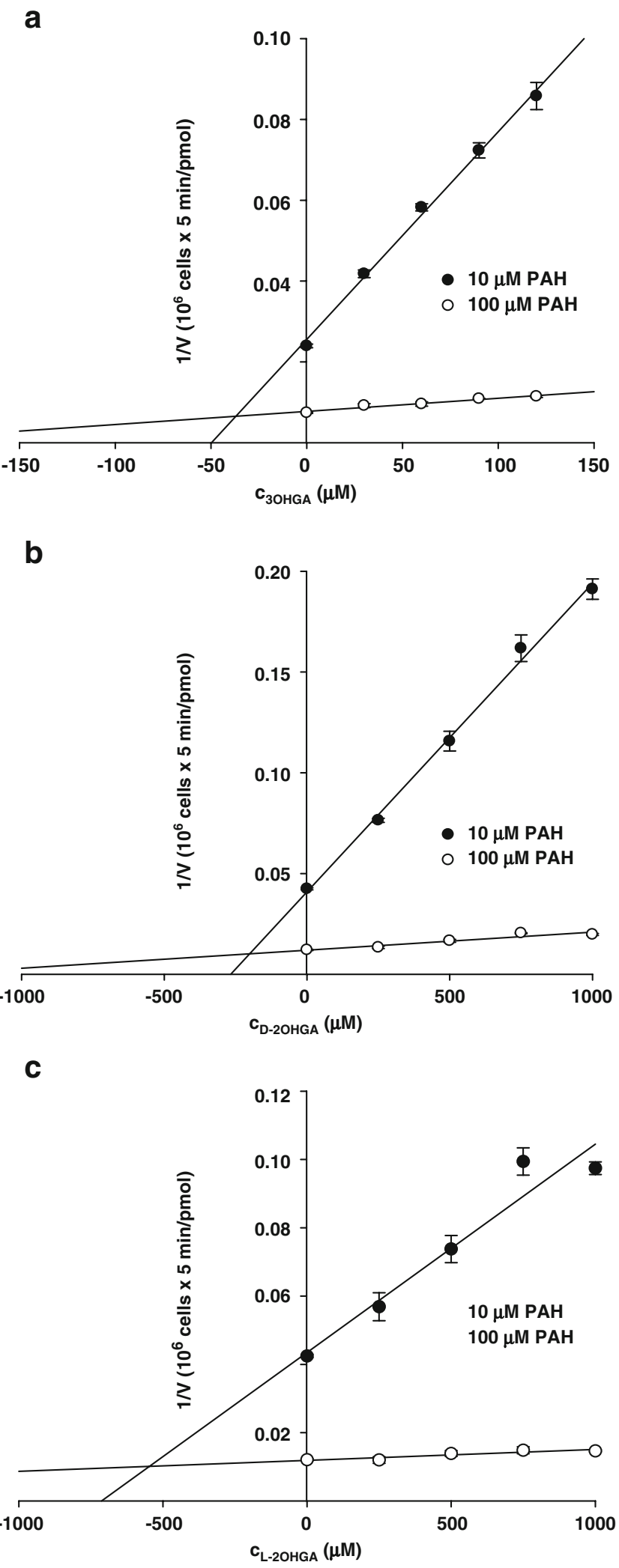

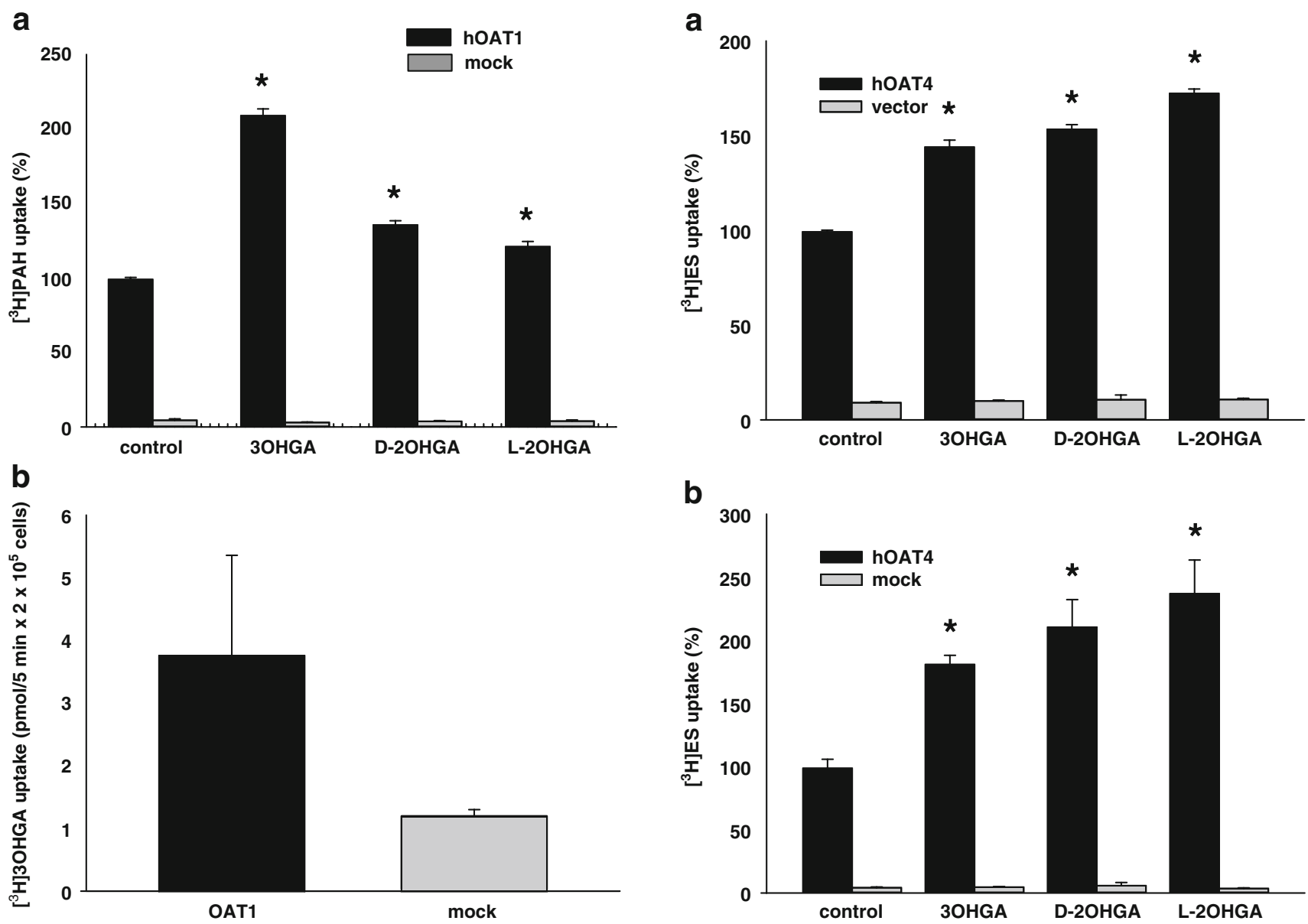

Fig. 5 Trans-stimulation of OAT1-mediated uptake by $\left[{ }^{3} \mathrm{H}\right] \mathrm{PAH}$ and direct evidence for $\left[{ }^{3} \mathrm{H}\right] 3 \mathrm{OHGA}$ uptake by OAT1. Trans-stimulation studies were performed by preloading OAT1-transfected HEK293 cells for $2 \mathrm{~h}$ with $1 \mathrm{mM}$ of 3OHGA, D-, or L-2OHGA (a). Subsequently, OAT1-mediated PAH-uptake $\left(1.2 \mu \mathrm{M}\left[{ }^{3} \mathrm{H}\right] \mathrm{PAH}\right.$ plus $8.8 \mu \mathrm{M}$ unlabeled PAH) was measured. Black columns represent the uptake measured in OAT1 and gray columns such measured in vectortransfected cells. The values represent means \pm SEM of four independent repeats. Significance was calculated by Students $t$-test, ${ }^{*} p<0.01$. In (b), the uptake of $20 \mathrm{nM}\left[{ }^{3} \mathrm{H}\right] \mathrm{OHGA}$ in hNaDC3-transfected HEK293 cells and mocks is compared. Data represent mean values of three determinations, where the uptake of $\left[{ }^{3} \mathrm{H}\right] \mathrm{OHGA}$ in hOAT1transfected cells of each individual experiments was set to $100 \%$

D-, and L-2OHGA) appeared to be better tolerated than a substitution at C3 (3OHGA). The two dicarboxylates with the lowest affinities (3OHGA, GC) exhibited a potentialdependent $K_{\mathrm{m}}$, whereas unsubstituted and $\mathrm{C} 2$-substituted dicarboxylates showed $K_{\mathrm{m}}$ values independent of membrane potential between -90 and $-30 \mathrm{mV}$. The heavy metal chelator, 2,3-dimercaptosuccinate, also had a $K_{\mathrm{m}}$ increasing with depolarization [22], suggesting that $\mathrm{hNaDC} 3$ has to perform a voltage-dependent step in interacting with compounds carrying a substitution at $\mathrm{C} 3$ or a double bond between $\mathrm{C} 2$ und $\mathrm{C} 3$. The maximal currents, $I_{\max }$, decreased in the order $\mathrm{D}-2 \mathrm{OHGA}>3 \mathrm{OHGA}=\mathrm{L}-2 \mathrm{OHGA}>\mathrm{GA}=$ adipate $>$

Fig. 6 Trans-stimulation of OAT4-mediated uptake by $\left[{ }^{3} \mathrm{H}\right]$ estrone sulfate. Trans-stimulation studies were performed by preloading (a) OAT4-expressing HEK293 cells for $2 \mathrm{~h}$ with $1 \mathrm{mM}$ of the indicated effectors or (b) OAT4-expressing oocytes by injecting $23 \mathrm{nl}$ of either $60 \mathrm{mM} 3 \mathrm{OHGA}$, D-, or L-2OHGA or an equivalent amount of ORi (control). Subsequently, OAT4-mediated $20 \mathrm{nM}\left[{ }^{3} \mathrm{H}\right] \mathrm{ES}$ uptake was examined. The gray columns represent the uptake in vector-transfected HEK293 cells (a) or mock-transfected oocytes (b). Black columns represent uptake of radioactively labeled substrates by OAT4expressing HEK293 cells (a) or oocytes (b). All experiments were standardized by setting the control (Ringer solution) of each experiment to $100 \%$. The values represent the mean \pm SEM of three independent experiments with four repeats of cells or six to 15 oocytes each. Significance was calculated by Students $t$-test, ${ }^{*} p<0.01$

$\mathrm{GC}>\alpha \mathrm{KG}$. Thereby, the turnover rate of $\mathrm{hNaDC} 3$ with a preference for $\mathrm{OH}$ substitutions at $\mathrm{C} 2$ and $\mathrm{C} 3$ appears to follow a different sequence than the affinity. The transport efficiency, $I_{\max } / K_{\mathrm{m}}$, was the highest for adipate and GA, followed by $\mathrm{D}-2 \mathrm{OHGA}>\alpha \mathrm{KG}>\mathrm{L}-2 \mathrm{OHGA}>3 \mathrm{OHGA} » \mathrm{GC}$. Thus, hNaDC3 may play an important role in the renal secretion particularly of adipate, GA, and 2OHGAs, but not for 3OHGA and GC. A reabsorption of these compounds by $\mathrm{hNaDC1}$ located in the apical [14] is unlikely, because $\mathrm{NaDC} 1$ has a low affinity for C5 dicarboxylates ([23] and own unpublished results). 
$\mathrm{NaDC} 3$ is not only localized in the basolateral membrane of human and rat kidneys [24] but also in rat astrocytes $[25,26]$ where it mediates $\alpha \mathrm{KG}$-inhibitable transport of $\left[{ }^{14} \mathrm{C}\right] N$-acetyl-L-aspartate, a compound with a dicarboxylic acid like structure. It needs to be established which role $\mathrm{hNaDC} 3$ plays in the brain of affected patients.

The multispecific OATs may represent additional candidates for high affinity transporters of GA derivatives [1517]. In our studies, 3OHGA, D-, and L-2OHGA did not interact with hOAT3 expressed in HEK293 cells, while hOAT1 translocated $\left[{ }^{3} \mathrm{H}\right] 3 \mathrm{OHGA} .\left[{ }^{3} \mathrm{H}\right] \mathrm{PAH}$ uptake in HEK293 cells transfected with hOAT1 cDNA was markedly reduced by 3 OHGA, D-, L-2OHGA, and adipate in cisinhibition (competition) studies. Vive versa, $\left[{ }^{3} \mathrm{H}\right] \mathrm{PAH}$ uptake was increased by preloading these cells with 3OHGA and D-, or L-2OHGA indicating exchange of PAH with either 3OHGA, D-, or L-2OHGA. These observations provide the first evidence that OAT1 not only interacts with its natural substrate $\alpha \mathrm{KG}$ but also with glutarates possessing hydroxyl groups at position $\mathrm{C} 2$ or $\mathrm{C} 3$. All GA derivatives exhibited competitive inhibition with PAH. 3OHGA showed a $K_{\mathrm{i}}$ much lower than D- or L2OHGA, suggesting that a C3-substituted dicarboxylate may fit better into the binding pocket of hOAT1 than dicarboxylates with substitutions at $\mathrm{C} 2$. Glutamate and GC at a concentration of $0.1 \mathrm{mM}$ only weakly interacted with hOAT1 indicating that positively charged substitutions, the amino group in glutamate, or a double bound in the carbon backbone (GC) do not fulfill an ideal configuration for substrates of hOAT1. Acceptance of dicarboxylates by hOAT1 is not restricted to C5 carbon backbone dicarboxylates as demonstrated by inhibition of PAH uptake by adipate (C6). A higher chain length (pimelate; C7 and suberate; C8) is not suitable for transport (data not shown). Suberate, present in the urine of GA2 patients, has to be excreted by transporters different from OAT1.

3OHGA, D-, and L-2OHGA did not interact with OAT3. These substances also did not inhibit OAT4, but transstimulated ES uptake into OAT4-transfected cells or OAT4expressing oocytes. Recently, we have shown that OAT4 which is located at the apical membrane of human proximal tubule cells is working in an asymmetric mode: it accepts dicarboxylates only from the inside (cytosolic side), but not from the outside (urine side) [20]. One of the physiological functions of OAT4 is the reabsorption of estrone sulfate and urate from the filtrate in exchange for intracellular dicarboxylates. Here, we provide experimental evidence of an exchange between extracellular ES and intracellular 3OHGA, L-, and D-2OHGA mediated by OAT4.

Our observations describe for the first time the basic components of the renal secretion of cytotoxic metabolites accumulating in patients suffering from glutaric acidurias. These data are summarized in a cell model for the renal excretion of GA and GA derivatives in Fig. 7. GA and GA derivatives are taken up from the plasma across the basolateral membrane by $\mathrm{OAT} 1$ and $\mathrm{NaDC} 3$ and are secreted into the urine via OAT4. It is, however, likely that $\mathrm{NaDC} 3$ is not only directly involved in the uptake of 3OHGA and 2OHGAs but mediates the transfer of $\alpha \mathrm{KG}$ into proximal tubule cells to supply anionic exchange equivalents for OAT1. The coordinated action of these transporters may guarantee a high renal clearance of GA and GA derivatives under metabolically stable conditions. In states of fever, however, downregulation of the expression of OAT1 [27] may partially impair renal secretion and cause accumulation of GA derivatives with possible side effects.
Fig. 7 Cell model for the renal secretion of GA derivatives. GA derivatives are taken up from the plasma by OAT1 and NaDC3 across the basolateral membrane and exit the cell by OAT4 in exchange for estrone sulfate or the more physiological anion chloride. The gradient required for sodium-dependent uptake via $\mathrm{NaDC} 3$ is maintained by the $\mathrm{Na}^{+} / \mathrm{K}^{+}$-ATPase

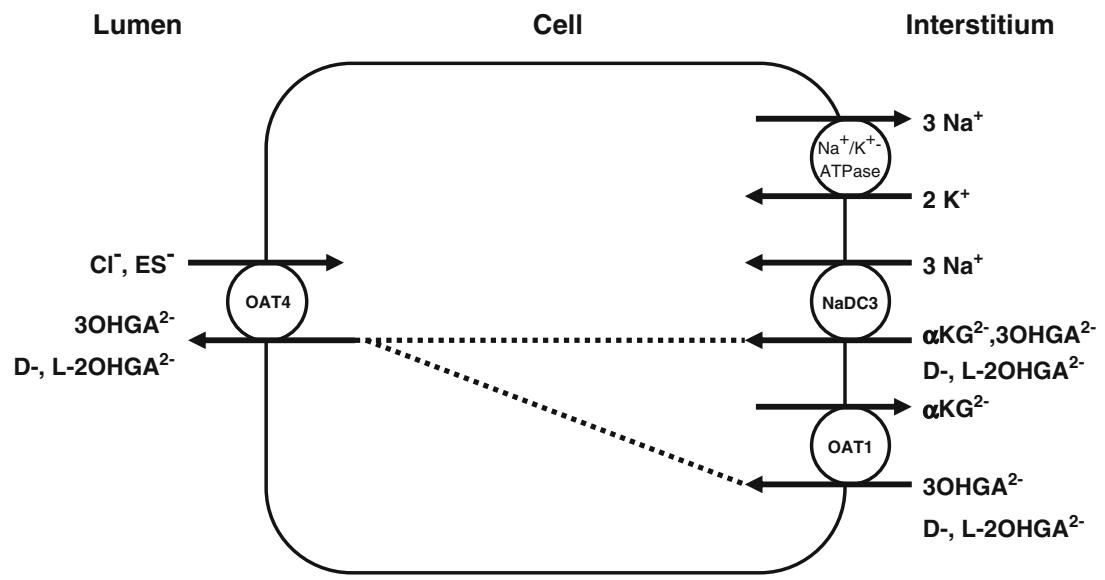


Acknowledgments This work was supported by the Else KrönerFresenius-Stiftung (P22/07 // A19/07) to B.C.B., by Deutsche Forschungsgemeinschaft (DFG grant MU $1778 / 2-1$ to C.M.), and by Arbeitsgemeinschaft für Pädiatrische Stoffwechselstörungen (APS) to C.M. We thank I. Markmann and S. Petzke for the technical assistance.

Open Access This article is distributed under the terms of the Creative Commons Attribution Noncommercial License which permits any noncommercial use, distribution, and reproduction in any medium, provided the original author(s) and source are credited.

\section{References}

1. Goodman SI, Frerman FE (2001) Organic acidemias due to defects in lysine oxidation: 2-ketoadipic acidemia and glutaric academia. In: Scriver CR, Beaudet AL, Sly WS, Valle D, Childs B, Kinzler KW, Vogelstein B (eds) The metabolic and molecular bases of inherited disease. McGraw-Hill Inc., New York, USA, pp 2195-2204

2. Hedlund GL, Longo N, Pasquali M (2006) Glutaric academia type 1. Am J Med Genet 142C:86-94

3. Strauss KA, Puffenberger EG, Robinson DL, Morton DH (2003) Type I glutaric aciduria, part 1: natural history of 77 patients. Am J Med Genet 121C:38-52

4. Kölker S, Koeller DM, Okun JG, Hoffmann GF (2004) Pathomechanisms of neurodegeneration in glutaryl-CoA dehydrogenase deficiency. Ann Neurol 55:7-12

5. Kölker S, Garbade SF, Greenberg CR, Leonard JV, Saudubray JM, Kalkanoglu HS, Lund AM, Merinero B, Wajner M, Troncoso $\mathrm{M}$, Williams $\mathrm{M}$, Walter $\mathrm{JH}$, Campistol $\mathrm{J}$, Marti-Herrero $\mathrm{M}$, Caswill M, Burlina AB, Lagler F, Maier EM, Schwahn B, Tokatli A, Dursun A, Coskun T, Chalmers RA, Koeller DM, Zschocke J, Christensen E, Burgard P, Hoffmann GF (2006) Natural history, outcome, and treatment efficacy in children and adults with glutaryl-CoA dehydrogenase deficiency. Pediatr Res 59:840-847

6. Przyrembel H, Wendel U, Becker K, Bremer HJ, Lieneke B, Ketting D, Wadman SK (1976) Glutaric aciduria type II: report on a previously undescribed metabolic disorder. Clin Chim Acta 66:227-239

7. Seijo-Martinez M, Navarro C, Castro del Rio M, Vila O, Puig M, Ribes A, Butron M (2005) L-2-Hydroxyglutaric aciduria: Clinical, neuroimaging, and neuropathological findings. Arch Neurol 62:666-670

8. Struys EA, Salomons GS, Achouri Y, Van Schaftingen E, Grosso S, Craigen WJ, Verhoeven NM, Jakobs C (2005) Mutations in the D-2-hydroxyglutarate dehydrogenase gene cause D-2-hydroxyglutaric aciduria. Am J Hum Genet 76:358-360

9. Struys EA (2006) D-2-hydroxyglutaric aciduria: unravelling the biochemical pathways and the genetic defect. J Inherit Metab Dis 29:21-29

10. Rzem R, Veiga-da-Cunha M, Noel G, Goffette S, Nassogne M-C, Tabarki B, Schöller C, Marquardt T, Vikkula M, Van Schaftingen E (2004) A gene encoding a putative FAD-dependent L-2hydroxyglutarate dehydrogenase is mutated in L-2-hydroxyglutaric aciduria. Proc Natl Acad Sci 101:16849-16854

11. Lindner M, Kölker S, Schulze A, Christensen E, Greenberg CR, Hoffmann GF (2004) Neonatal screening for glutaryl-CoA dehydrogenase deficiency. J Inherit Metab Dis 27:851-859

12. Burckhardt BC, Lorenz J, Kobbe C, Burckhardt G (2004) Substrate specificity of the human renal sodium dicarboxylate cotransporter, hNaDC-3, under voltage clamp conditions. Am J Physiol Renal Physiol 288:792-799

13. Stellmer F, Keyser B, Burckhardt BC, Koepsell H, Streichert T, Glatzel M, Jabs S, Thiem J, Herdering W, Koeller DM, Goodman SI, Lukacs Z, Ullrich K, Burckhardt G, Braulke T, Mühlhausen C (2007) 3-Hydroxglutaric acid is transported via the sodiumdependent dicarboxylate transporter NaDC3. J Mol Med 65:763-770

14. Pajor AM (2006) Molecular properties of the slc13 family of dicarboxylate and sulfate transporters. Pflugers Arch-Eur J Physiol 451:597-605

15. Rizwan AN, Burckhardt G (2007) Organic anion transporters of the SLC22 family: Biopharmaceutical, physiological, and pathological roles. Pharm Res 24:450-470

16. Anzai N, Kanai Y, Endou H (2006) Organic anion transporter family: current knowledge. J Pharmacol Sci 100:411-426

17. Robertson EE, Rankin GO (2006) Human renal organic anion transporters: characteristics and contributions to drug and drug metabolite excretion. Pharmacol Ther 109:399-412

18. Alebouyeh M, Takeda M, Onozato ML, Tojo A, Noshiro R, Hasannejad H, Inatomi J, Narikawa S, Huang X-L, Khamdang S, Anzai N, Endou H (2003) Expression of human organic anion transporters in the choroid plexus and their interactions with neurotransmitter metabolites. J Pharmacol Sci 93:430-436

19. Bakhiya N, Stephani M, Bahn A, Ugele B, Seidel A, Burckhardt G, Glatt H (2006) Uptake of chemically reactive, DNA-damaging sulfuric acid esters into renal cells by human organic anion transporters. J Am Soc Nephrol 17:1414-1421

20. Hagos Y, Stein D, Ugele B, Burckhardt G, Bahn A (2007) Human renal organic anion transporter 4 operates as an asymmetric urate transporter. J Am Soc Nephrol 18:430-439

21. Mühlhausen C, Ott N, Chalajour F, Tilki D, Freudenberg F, Shahhossini M, Thiem J, Ullrich K, Braulke T, Ergün S (2006) Endothelial effects of 3-hydroxyglutaric acid: implications for glutaric aciduria type I. Pediatr Res 59:196-202

22. Burckhardt BC, Drinkuth B, Menzel C, König A, Steffgen J, Wright SH, Burckhardt $\mathrm{G}$ (2002) The renal $\mathrm{Na}^{+}$-dependent dicarboxylate transporter, $\mathrm{NaDC} 3$, translocates dimethyl- and disulfhydryl-compounds and contributes to renal heavy metal detoxification. J Am Soc Nephrol 13:2628-2638

23. Pajor AM, Sun N (1996) Functional differences between rabbit and human $\mathrm{Na}^{+}$-dicarboxylate cotransporters, NaDC-1 and hNaDC-1. Am J Physiol Renal Physiol 271:F1093-F1099

24. Wang J, Chen X, Zhu H, Peng L, Hong Q (2003) Relationship between aging and renal high-affinity sodium-dependent dicarboxylate cotransporter-3 expression characterized with antifusion protein antibody. J Geront 58A:879-888

25. Yodoya E, Wada M, Shimada A, Katsukawa H, Okada N, Yamamoto A, Ganapathy V, Fujita T (2005) Functional and molecular identification of sodium-coupled dicarboxylate transporters in rat primary cultured cerebrocortical astrocytes and neurons. J Neurochem 97:162-173

26. Fujita T, Katsukawa H, Yodoya E, Wada M, Shimada A, Okada N, Yamamoto A, Ganapathy V (2005) Transport characteristics of $\mathrm{N}$-acetyl-L-aspartate in rat astrocytes: involvement of sodiumcoupled high-affinity carboxylate transporter $\mathrm{NaC} 3 / \mathrm{NaDC} 3$-mediated transport. J Neurochem 93:706-714

27. Sauvant C, Holzinger H, Gekle M (2006) Prostaglandin $E_{2}$ inhibits its own renal transport by downregulation of organic anion transporters rOAT1 and rOAT3. J Am Soc Nephrol 17: $46-53$ 